\title{
EDUCAÇÃO ALIMENTAR E NUTRICIONAL COMO INTERVENÇÃO EM HÁBITOS ALIMENTARES SAUDÁVEIS NO AMBIENTE ESCOLAR
}

FOOD AND NUTRITION EDUCATION AS AN INTERVENTION IN HEALTHY EATING HABITS IN THE SCHOOL ENVIRONMENT

EDUCACIÓN ALIMENTARIA Y NUTRICIONAL COMO INTERVENCIÓN EN HÁBITOS ALIMENTARIOS SALUdABLES EN EL AMBIENTE

ESCOLAR

Quitéria Vanessa Brito Magalhães ${ }^{1}$

Jorge Luís Pereira Cavalcante ${ }^{2}$

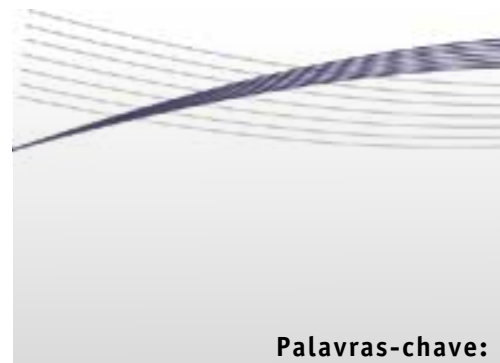

Educação Alimentar e Nutricional; Hábitos Alimentares; Saúde Escolar.

Keywords:

Food and Nutrition Education; Eating Habits; School Health.

Palabras clave: Educación Alimentaria y Nutricional; Hábitos Alimentarios; Salud Escolar.

Submetido: 02/09/2018 Aprovado: 20/02/2019

Autor(a) para Correspondência: Quitéria Vanessa Brito Magalhães R. Inácio Alves de Mesquita, 578 Piracicaba - Santa Quitéria (CE) CEP: 62280-000 E-mail:vanessanutriris@gmail.com

\section{RESUMO}

Este estudo teve por objetivo analisar na literatura a influência das ações de educação alimentar e nutricional (EAN) como intervenção em hábitos alimentares no ambiente escolar. Trata-se de revisão integrativa, realizada com 10 artigos originais publicados em português no período de 2008 a 2017, no Brasil, disponíveis nas bases de dados Lilacs, MedLine e SciELO. Os artigos selecionados foram catalogados e identificados por título, autor, base de dados, ano de publicação, local, metodologia, objetivo do estudo, resultados e conclusões. A pesquisa mostrou similaridade de pensamento entre os autores ao identificar a EAN como estratégia que deve ser amplamente empregada no ambiente escolar com vistas a influenciar as escolhas alimentares e a formação de hábitos alimentares saudáveis. Constatou-se a necessidade de conduzir novas pesquisas para atualização dos dados disponíveis na literatura, relativas a ações de EAN voltadas aos escolares, em busca da construção de hábitos alimentares saudáveis e da melhoria da qualidade de vida.

1. Nutricionista. Especialista em Saúde da Família e Comunidade pela Escola de Saúde Pública do Ceará (ESP/ CE). Santa Quitéria (CE), Brasil.

2. Nutricionista. Aluno de Doutorado em Nutrição na Universidade Internacional Iberoamericana (UNINI México). Professor no Curso de Bacharelado em Nutrição do Centro Universitário Inta (UnInta). Sobral (CE), Brasil. 


\section{ABSTRACT}

This study aimed to analyze in the literature the influence of food and nutrition education (FNE) actions as an intervention in eating habits in the school environment. This is an integrative review, carried out with 10 original articles published in Portuguese within the period from 2008 to 2017, in Brazil, available on the databases LILACS, MedLine, and SciELO. The selected articles were cataloged and identified by title, author, database, year of publication, location, methodology, study objective, results, and conclusions. The research showed a similarity of thought between the authors when identifying FNE as a strategy that must be widely used in the school environment with a view to influencing food choices and the adoption of healthy eating habits. There was a need to conduct further research to update the data available in the literature, regarding FNE actions aimed at school students, seeking the construction of healthy eating habits and an improved quality of life.

\section{RESUMEN}

Este estudio tuvo como objetivo analizar en la literatura la influencia de acciones de educación alimentaria y nutricional (EAN) como una intervención en los hábitos alimentarios en el ambiente escolar. Esta es una revisión integradora, realizada con 10 artículos originales publicados en portugués en el período de 2008 a 2017, en Brasil, disponibles en las bases de datos LILACS, MedLine y SciELO. Los artículos seleccionados fueron catalogados e identificados por título, autor, base de datos, año de publicación, lugar, metodología, objetivo del estudio, resultados y conclusiones. La investigación mostró una similitud de pensamiento entre los autores cuando identificaron la EAN como una estrategia que debe ser ampliamente utilizada en el ambiente escolar con el fin de influir en la elección de alimentos y la adopción de hábitos alimentarios saludables. Se constató la necesidad de conducir nuevas investigaciones para actualizar los datos disponibles en la literatura, relativas a acciones de EAN dirigidas a los escolares, en busca de la construcción de hábitos alimentarios saludables y de la mejora de la calidad de vida.

\section{INTRODUÇÃ $O$}

0 quadro epidemiológico nutricional da população brasileira advém da coexistência de carências nutricionais e doenças infecciosas, bem como de um gradativo aumento dos índices de obesidade/ sobrepeso e de doenças crônicas não transmissíveis em todas as faixas etárias ${ }^{1}$. A obesidade/sobrepeso tem-se revelado um grande desafio para a saúde pública mundial, sendo que nas últimas três décadas suas taxas de incidência e prevalência apresentaram um aumento significativo, confirmando os dados da Organização Mundial da Saúde $(0 M S)^{2}$. A obesidade, inclusive em crianças e adolescentes, configura um dos maiores problemas de saúde pública e suas causas são multifatoriais: a) consumo excessivo e/ou desbalanceado de alimentos; b) condições socioeconômicas e culturais; c) sedentarismo; e d) contexto de insegurança alimentar e nutricional ${ }^{3}$.

No Brasil, a insegurança alimentar e nutricional foi avaliada pela Pesquisa Nacional por Amostra em Domicílios (PNAD), em 2013. Constatou-se que 52 milhões de pessoas $(25,8 \%)$ vivenciavam a insegurança alimentar - a maioria destas morava em zona urbana (40 milhões) e o Nordeste apresentou o maior número de domicílios em contexto de insegurança alimentar $(44,2 \%)^{4}$.
Considerando o aumento da incidência da obesidade, tem-se fomentado discussões sobre a adoção de ações em saúde no ambiente escolar, com destaque para a educação alimentar e nutricional (EAN). É importante entender que essas condutas buscam reverter o quadro de excesso de peso proporcionando meios para alcançar um peso ideal e uma alimentação e nutrição adequada, em especial junto à criança em idade escolar ${ }^{5}$. Nesse sentido, a EAN constitui uma estratégia que incorpora ações de prevenção de agravos, promoção da saúde e controle de problemas alimentares e nutricionais - como obesidade, doenças crônicas não transmissíveis e deficiências nutricionais ${ }^{6}$.

Um estudo ${ }^{7}$ apontou ser a infância o período de formação dos hábitos nutricionais da vida adulta, assim, trata-se da fase decisiva para estabelecer as bases de uma alimentação adequada e saudável. Nesse sentido, os autores de outro estudo ${ }^{8}$ afirmam que as instituições escolares, em especial as creches, exercem grande influência nos hábitos alimentares em decorrência do longo período de permanência das crianças no espaço educacional. É nesse contexto que a EAN se consolida como modo de reverter o quadro de obesidade via mudança de hábitos alimentares, devendo abranger todas as faixas etárias da educação formal ${ }^{9}$. 
Mostra-se necessário adotar ações de EAN baseadas em diferentes abordagens educacionais e pedagógicas, favorecendo o diálogo e a reflexão sobre aspectos da alimentação, nutrição e saúde ${ }^{10}$. Logo, a escola proporciona um espaço indispensável às ações de EAN, promovendo hábitos alimentares saudáveis que visam à promoção da saúde. A oferta de uma alimentação equilibrada e o estímulo à adoção de bons hábitos alimentares no ambiente escolar são fundamentais para minimizar a insegurança alimentar e nutricional e auxiliar o aluno a garantir seu bemestar e sua qualidade de vida, com capacidade satisfatória de aprendizado e desenvolvimento ${ }^{7}$.

De acordo com a Política Nacional de Alimentação e Nutrição (PNAN), as ações de alimentação e nutrição devem ser concomitantes às de saúde, em caráter complementar, com formulação, execução e avaliação no âmbito do Sistema Único de Saúde (SUS) ${ }^{6}$. Assim, a EAN assume importante papel na promoção de hábitos alimentares saudáveis e proporciona conhecimentos importantes para a tomada de decisões nesse sentido ${ }^{7}$.

0 uso de ferramentas como a EAN constitui um desafio tanto para os nutricionistas e professores quanto para a população, além de estabelecer ações de extrema importância. Diante do cenário observado, levantou-se a seguinte questão de pesquisa:

- Como as ações de educação alimentar podem influenciar os hábitos alimentares de escolares?

Logo, para ampliar as discussões relativas ao tema, este estudo teve por objetivo:

- Analisar na literatura a influência das ações de educação alimentar e nutricional como intervenção em hábitos alimentares no ambiente escolar.

\section{METODOLOGIA}

Trata-se de revisão integrativa de literatura, baseada em artigos disponíveis nas bases de dados Lilacs, MedLine e SciELO. Os critérios de inclusão foram: a) artigos originais; b) estudos produzidos no Brasil; c) publicações em língua portuguesa; e d) datas de publicação entre 2008 e 2017. Já os critérios de exclusão foram: a) artigos de revisão; e b) estudos científicos indisponíveis em formato de artigo (monografias, dissertações e teses).

A busca de artigos ocorreu entre junho de 2016 e maio de 2017, com uso dos seguintes descritores em ciências da saúde (DeCS), de modo isolado ou cruzado: a) "educação alimentar e nutricional"; b) "hábitos alimentares"; e c) "saúde escolar". 0 cruzamento dessas palavras-chave se deu da seguinte forma: a) "educação alimentar e nutricional versus saúde escolar"; b) "hábitos alimentares versus saúde escolar"; e c) "educação alimentar e nutricional versus hábitos alimentares".

A aplicação dos critérios de inclusão resultou na seleção de 23 dos 47 artigos identificados. Esses 23 artigos foram submetidos a leitura integral e 13 deles foram excluídos por não estarem plenamente de acordo com a temática em análise, além de não serem artigos originais. Assim, a amostra final desta revisão integrativa consistiu em 10 artigos catalogados e identificados por título, autor, base de dados, ano de publicação, local, metodologia, objetivo do estudo, resultados e conclusões. Posteriormente, estabeleceu-se um paralelo entre os estudos, interpretando e interconectando os achados de pesquisa e apresentando-os sob a forma de sintese do conhecimento.

Este estudo se manteve fiel às opiniões dos autores das obras consultadas e respeitou suas definições, seus conceitos, suas ideias e seus princípios.

\section{RESULTADOS}

0 Quadro 1 sintetiza os 10 artigos incluídos na amostra final desta revisão integrativa (5 selecionados na base Lilacs, 1 na base MedLine e 4 na base SciELO. 


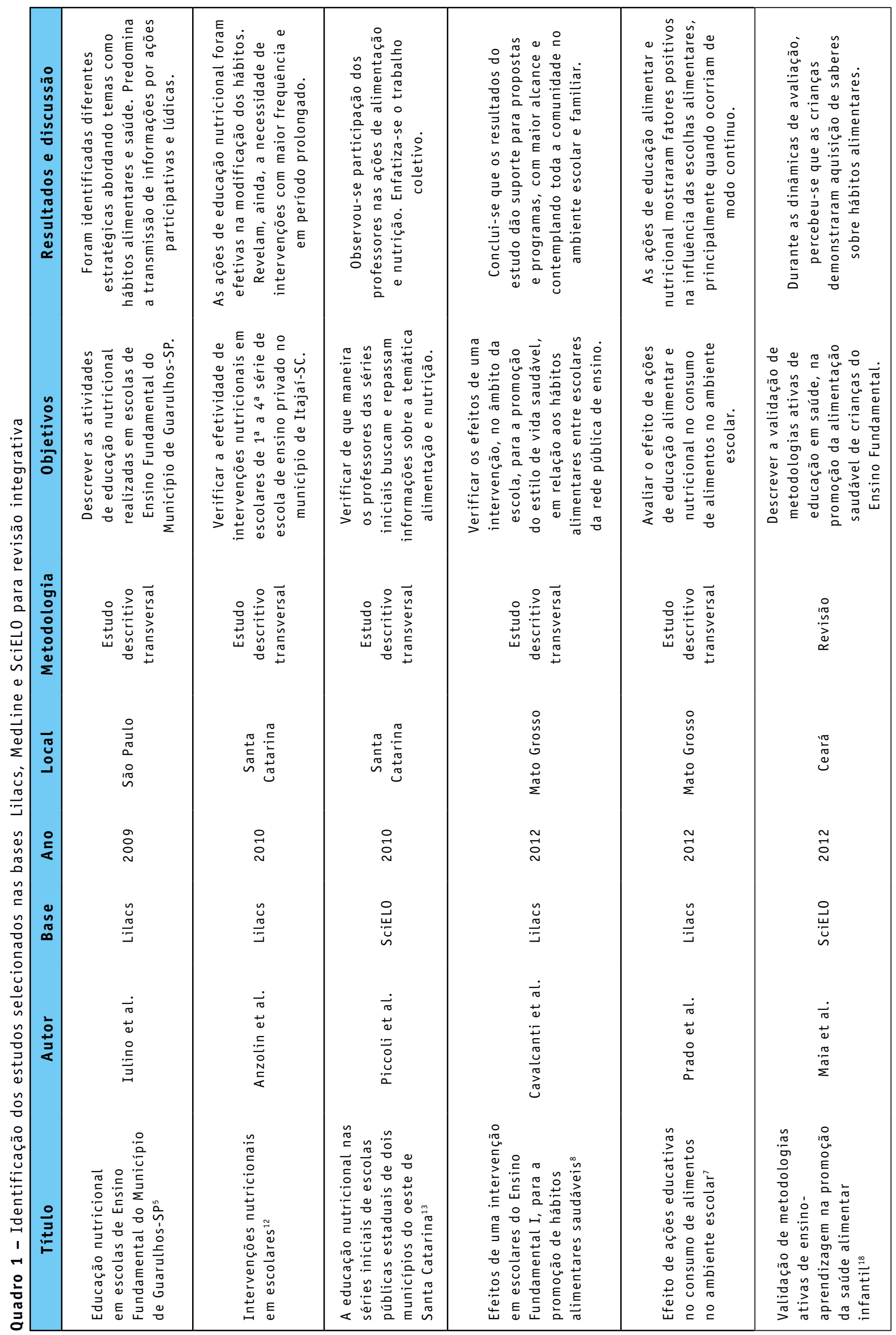




\begin{tabular}{|c|c|c|c|c|}
\hline 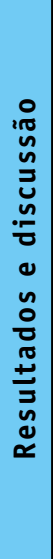 & 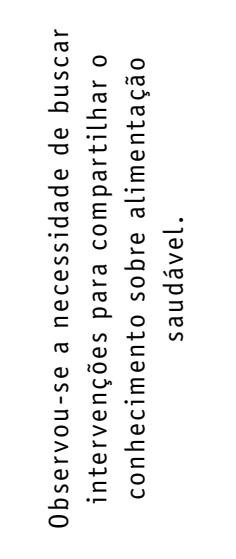 & 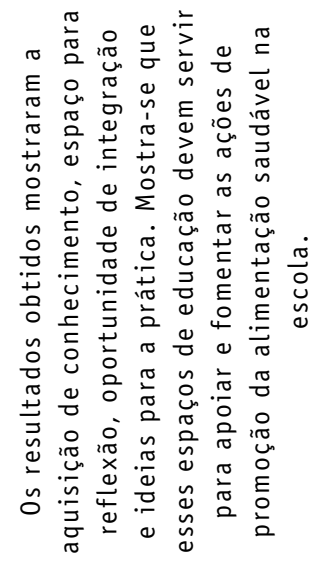 & 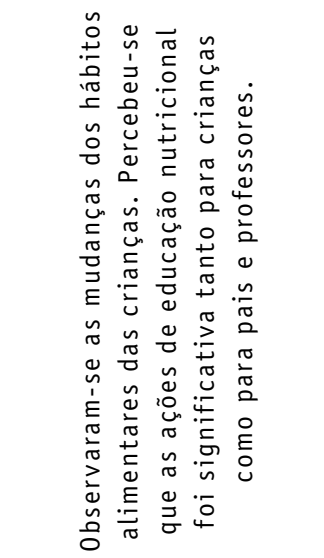 & 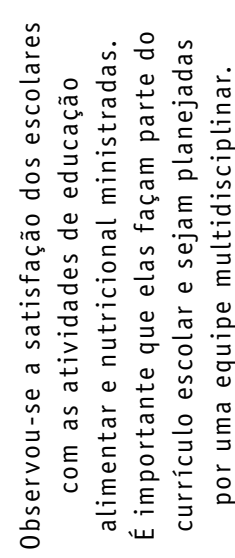 \\
\hline 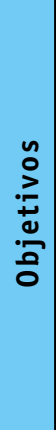 & 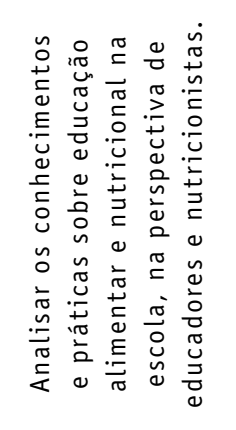 & 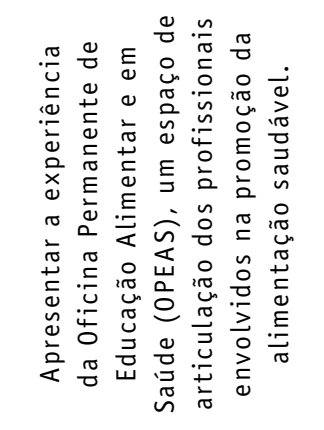 & 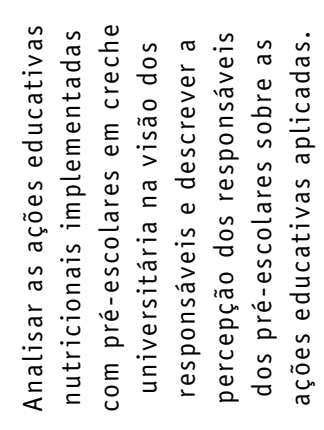 & 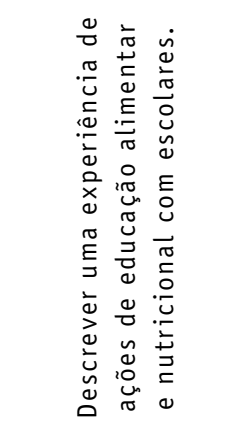 \\
\hline $\begin{array}{l}\frac{\pi}{5} \\
\frac{0}{0} \\
0 \\
0 \\
\frac{0}{0} \\
\frac{0}{2}\end{array}$ & 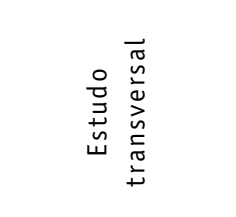 & 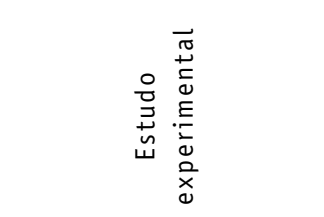 & 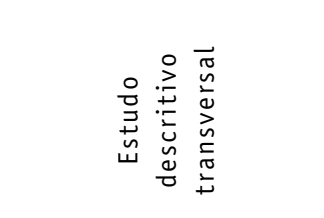 & 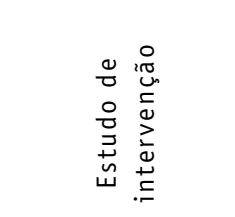 \\
\hline $\begin{array}{l}\bar{\Xi} \\
\text { ర్ }\end{array}$ & 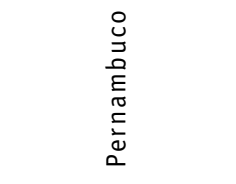 & 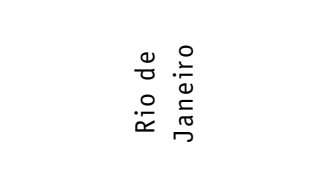 & 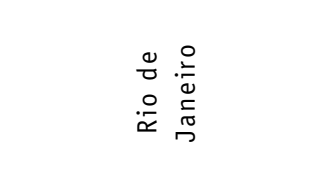 & $\begin{array}{l}0 \\
\tilde{n} \\
\stackrel{0}{0} \\
0 \\
0 \\
D^{\pi}\end{array}$ \\
\hline$\stackrel{\circ}{\stackrel{一}{\leftarrow}}$ & $\stackrel{m}{\stackrel{n}{a}}$ & $\stackrel{m}{\stackrel{m}{\sim}}$ & $\underset{\sim}{\stackrel{\sim}{\sim}}$ & $\begin{array}{l}\stackrel{0}{\circ} \\
\stackrel{\sim}{n}\end{array}$ \\
\hline ఐ & 号 & 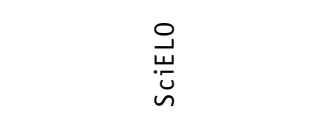 & 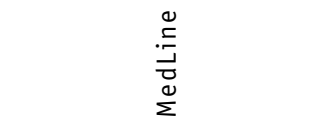 & $\begin{array}{l}\stackrel{\widetilde{\sigma}}{:} \\
:\end{array}$ \\
\hline 京 & 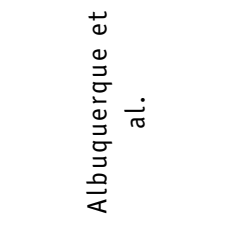 & 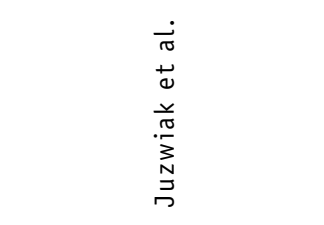 & 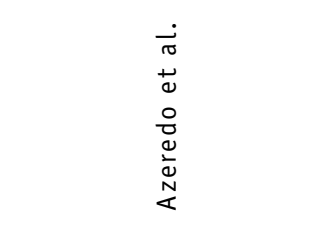 & $\begin{array}{l}\dot{0} \\
+ \\
+ \\
0 \\
\stackrel{0}{0} \\
\frac{\pi}{2}\end{array}$ \\
\hline 总 & 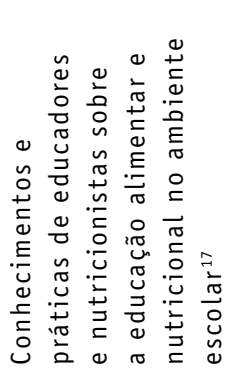 & 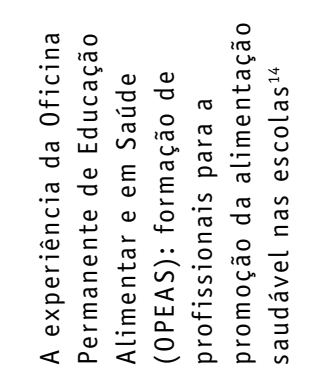 & 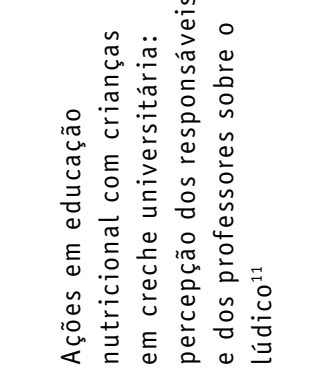 & 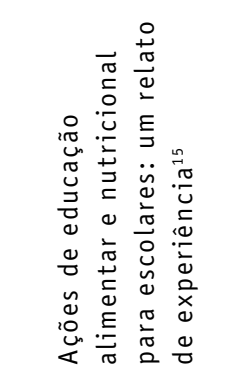 \\
\hline
\end{tabular}


Como se observa no Quadro 1, inicialmente em relação ao ano de publicação, vale destacar que $90 \%$ dos estudos selecionados foram publicados de 2010 a 2017 e $70 \%$ nos últimos 5 anos do período. Além disso, $60 \%$ dos artigos selecionados foram publicados em periódicos da área de Nutrição e $40 \%$ em periódicos de Saúde Pública e Coletiva.

A maioria dos estudos foi publicada no CentroSul do Brasil - 30\% em Mato Grosso, 20\% em Santa Catarina, $20 \%$ no Rio de Janeiro e $10 \%$ em São Paulo. No entanto, quanto à metodologia adotada, predominaram os estudos de intervenção $(90 \%)$ e os relatos de experiência (10\%).

Finalmente, observou-se baixo número de publicações sobre ações de EAN com escolares na promoção de hábitos alimentares saudáveis. Por isso, mostra-se necessário conduzir novos estudos voltados a essa temática e esse público para aprimorar o conhecimento sobre o assunto.

\section{DISCUSSÃO}

A maioria dos autores reforça a importância das ações de EAN na modificação dos hábitos alimentares de escolares no decorrer do estudo ${ }^{11,12}$. Outros autores mencionam que a pesquisa voltada à eficácia de ações de EAN apresentou dificuldades, pois grande parte dos autores menciona a escassez de estudos sobre o tema. Assim, faz-se necessário realizar novos estudos que esclareçam as ações relativas a alimentação e nutriçã $0^{13}$.

De todo modo, os artigos selecionados possibilitaram apontar que a intervenção nutricional por meio da EAN influencia os hábitos alimentares saudáveis, especialmente quando abordada de modo continuado no ambiente escolar - espaço favorável para os escolares adotarem uma alimentação saudável?.

Como registra o Quadro 1, a EAN tem sido a atividade de promoção da saúde mais frequente nas escolas de acordo com profissionais das mais diversas áreas da saúde ${ }^{14}$. Contudo, a EAN compreende a transmissão de conhecimentos relativos à promoção dos hábitos alimentares entre os escolares, considerando a interdisciplinaridade dos pesquisadores e da equipe multidisciplinar, que modifica o comportamento dos escolares diante dos hábitos alimentares saudáveis ${ }^{15}$.

Alguns estudos indicam uma relação entre 0 aumento do índice de obesidade e os estados mais desenvolvidos do Brasil, com maior abrangência em

\section{...a EAN tem sido a atividade de promoção da saúde mais frequente nas escolas...}

estados do Centro-Sul ${ }^{8,15}$. Um estudo ${ }^{7}$ com intervenção junto a escolares de 8 a 14 anos de 2 escolas públicas de Cuiabá-MT avaliou o efeito de ações de EAN no consumo de alimentos no ambiente escolar - essa intervenção identificou um efeito positivo nas escolhas alimentares saudáveis, indicando que o consumo de alimentos em cantinas escolares e estabelecimentos comerciais nas proximidades da escola constitui uma preocupação de EAN. Ou seja, os hábitos alimentares precisam mudar, pois os escolares consomem o que se encontra disponivel e, normalmente, cantinas escolares e as chamadas "vendinhas" comercializam alimentos com alta densidade calórica, ricos em gorduras e açúcares.

Outros estudos realizados com escolares ${ }^{8,12}$ corroboram que os alimentos mais adquiridos pelos escolares são doces, refrigerantes e salgadinhos. Observou-se, ainda, alto consumo de merenda escolar pela maioria dos escolares, que se mostra mais saudável, porém, sua preferência recai sobre os produtos disponíveis em cantinas e vendinhas considerados mais saborosos ${ }^{12}$.

Observaram-se melhorias em relação à qualidade dos alimentos consumidos pelos escolares após ações de $E N^{13}$ e os professores vêm trabalhando a temática da alimentação saudável, mas indicam a necessidade de recorrer à EAN de modo coletivo, junto com outros profissionais.

A promoção de atividades de EAN nas escolas (como orientações, jogos, palestras, atividades lúdicas e criação de hortas) deve ser fruto de ação conjunta de educadores e nutricionistas, que passam a ser responsáveis pela intermediação de saberes e assumem papéis determinantes na adoção de hábitos alimentares saudáveis na escola ${ }^{5}$. Acrescenta-se que os pais são os primeiros agentes a lidar com as práticas alimentares dos escolares ${ }^{11}$.

A EAN é um campo de ação da segurança alimentar e nutricional (SAN), que almeja auxiliar nas mudanças alimentares pessoais e da sociedade ${ }^{5,7,12}$. Comenta-se, ainda, que a EAN tem sido considerada 
uma estratégia para construção do conhecimento e motivação para a alimentação saudável, a promoção de saúde e a SAN, destacando-se, também, o controle dos problemas alimentares e nutricionais contemporâneos.

Outros estudos ${ }^{7,11}$ consideram as ações de EAN fundamentais para implementar a prevenção primária no controle e na redução das taxas de sobrepeso e obesidade, além de outros distúrbios alimentares. 0s autores salientam que a escola deve trabalhar junto com todos envolvidos na educação para alcançar esses objetivos, firmando parcerias com os pais e a comunidade.

A inserção da EAN no currículo escolar, em consonância com os parâmetros curriculares nacionais (PCNs) e as diretrizes da PNAN, sugere que toda a escola integrada (profissionais da saúde e da educação, pais e alunos) realize ações diversas que favoreçam os princípios de promoção da saúde indicados pela $0 \mathrm{MS}^{6}$. A perspectiva da promoção da saúde se apresenta e é apontada como hábitos alimentares saudáveis, garantia da qualidade dos alimentos e prevenção de distúrbios nutricionais (desde a desnutrição até a obesidade ${ }^{16}$.

As intervenções de EAN devem ser disciplinares e envolventes, reforçando o esclarecimento de que o ensino sobre alimentação e nutrição nas escolas deve ser incentivado pelos órgãos públicos, mediante cursos de capacitação para os professores, de modo que estes sejam facilitadores das modificações no comportamento alimentar ${ }^{14}$.

Outro ponto a destacar é a realização das ações de EAN junto aos escolares com uso de vídeos, dinâmicas, antropometria e atividades lúdicas, como caça-palavras, jogo da memória, oficinas e teatro de fantoches. Já se mencionou que as estratégias educativas são fundamentais para a promoção da saúde, a conscientização quanto à prática de hábitos alimentares saudáveis, principalmente diante dos escolares, mas que refletiram em seus familiares e em toda comunidade escolar, proporcionando os conhecimentos necessários para mudar hábitos alimentares ${ }^{5,15}$.

Elaborar ou escolher uma metodologia visando a adotar ações de EAN e promover a saúde escolar constitui um desafio. Isso se dá, muitas vezes, porque os escolares já tinham concepções relativas a fatores psicológicos, familiares, sociais e econômicos ${ }^{17}$. As estratégicas metodológicas são importantes ferramentas para efetivar a prevenção de agravos e a promoção da saúde, principalmente em

\section{As intervenções \\ de EAN devem ser disciplinares \\ e envolventes...}

ambientes que possam estimular o compartilhamento de informações, o ensino e a aprendizagem no âmbito escolar ${ }^{15,18}$.

Esta pesquisa constatou que a implementação de ações de EAN como estratégia de intervenção em hábitos alimentares saudáveis vai além dos debates teóricos e ações de curto prazo. Isso foi citado em dois estudos ${ }^{7,15}$ que evidenciaram que as ações de EAN voltadas à alimentação saudável parecem influenciar as escolhas alimentares, especialmente em caráter continuado e rotineiro nessa etapa da vida em que se formam os hábitos alimentares. Na mesma linha de pensamento, outros estudos ${ }^{8,17}$ constataram que a EAN desempenha um importante papel no processo de transformação, recuperação e promoção de hábitos alimentares saudáveis, proporcionando os conhecimentos necessários para a tomada de decisões alimentares adequadas.

A maioria dos resultados das ações de EAN no âmbito escolar foi expressa por estudos de intervenção, observando-se similaridade entre as linhas de pensamento sobre a eficácia de estratégias de intervenção para a adoção de hábitos alimentares saudáveis entre escolares ${ }^{17}$. Quanto aos métodos de avaliação das intervenções, um estudo ${ }^{8}$ de avaliação nutricional com ações de educação nutricional e alimentar não apresentou efeito significativo na redução do índice de massa corporal (IMC) nas fases pré e pós-intervenção. Tal resultado sugere que o método de avaliação nutricional não é adequado para ações de curto prazo, pressupondo que estas sejam realizadas em longo prazo, ocasionando significativa redução do IMC.

Uma experiência de oficina permanente de EAN realizada com escolares reuniu várias classes de profissionais, como professores, gestores, equipes pedagógicas e nutricionistas. Constatou-se a sensibilização dos profissionais quanto à necessidade de entenderem que todos estão envolvidos na construção de conhecimentos voltados à promoção da alimentação saudável nas escolas, além do 
distanciamento entre alguns profissionais, o que dificulta as ações interprofissionais no ambiente escolar ${ }^{14}$. Nessa mesma linha de raciocínio, alguns estudos $5,9,17,19,20$ concordam quanto à necessidade de manter uma participação ativa entre todas as partes interessadas para alcançar a efetividade educativa em nutrição.

Desse modo, outros estudos ${ }^{7,17}$ enfatizam que, para a promoção da saúde, a EAN deve buscar ações nutricionais relativas tanto a hábitos alimentares saudáveis quanto à prevenção de doenças. Também se acredita na importância do ambiente escolar como cenário oportuno não só por promover ações educativas, mas para ampliar o acesso a uma alimentação saudável mediante estratégias de intervenção nutricional em hábitos alimentares.

\section{CONCLUSÃO}

0 uso da EAN como modo de intervenção, via ações e práticas, visa à promoção de hábitos alimentares saudáveis entre os escolares para o controle de obesidade e doenças crônicas não transmissíveis. Esses fatores se tornaram os problemas de saúde pública mais graves do século XXI em nível nacional e global.

A ampliação do conhecimento sobre nutrição, por parte de todos os profissionais que atuam nos campos da educação e da alimentação, mostrou a importância do empenho e da dedicação de todas as pessoas envolvidas na EAN junto aos escolares. Assume destaque a atuação dos pais e familiares nesses processos, proporcionando ações de longo prazo que potencializam os resultados na formação do comportamento alimentar das crianças.

Destaca-se que são notáveis as mudanças em termos de perfil alimentar, estilo de vida e padrão de saúde da população diante das ações de EAN postas em prática com escolares. Portanto, torna-se importante o uso de materiais lúdicos para as ações, a fim de esclarecer dúvidas e trocar experiências ao promover uma alimentação saudável.

Vale ressaltar que ainda há inúmeros caminhos a percorrer para consolidar a EAN no ambiente escolar, com pleno entendimento do papel do alimento e das mudanças no perfil alimentar, no estilo de vida e no padrão de saúde. Assim, espera-se que as ações sejam efetivas, contínuas e permanentes, possibilitando a redução dos índices epidemiológicos de obesidade.

Constatou-se a necessidade de realizar novas pesquisas acerca dos aspectos nutricionais de

\section{Assume destaque a atuação dos pais e familiares nesses processos...}

escolares. Isso possibilitará o direcionamento de metodologias de tratamento de fácil acesso; a melhora da qualidade de vida bem; e a atualização dos dados disponíveis na literatura. Como a quantidade de artigos sobre o tema ainda é considerada pequena diante dos danos que os hábitos alimentares causam aos escolares, novos achados de pesquisa tendem a apontar novos caminhos em busca de uma qualidade de vida estável por meio da alimentação saudável.

\section{CONTRIBUIÇÃO DOS AUTORES}

Quitéria Vanessa Brito Magalhães e Jorge Luís Pereira Cavalcante contribuíram com a realização da pesquisa, o delineamento do estudo e a redação e revisão crítica do manuscrito.

\section{REFERÊNCIAS}

1. Pimenta TAM, Rocha RA. Obesidade infantil no Brasil: um estudo comparativo entre a PNSN/1989 e a POF/200809 entre crianças de 5 a 9 anos de idade. FIEP Bulletin [serial on the internet]. 2012 [cited $2016 \mathrm{Jul} 12$ ];82(Spec):[about 5 pages]. Available from: $\quad$ http://www.fiepbulletin.net/index.php/ fiepbulletin/article/viewFile/2224/4310

2. Instituto Brasileiro de Geografia e Estatística. Pesquisa de orçamentos familiares 2008-2009: antropometria e estado nutricional de crianças, adolescentes e adultos no Brasil. Rio de Janeiro: IBGE; 2011.

3. Levy RB, Claro RM, Sichieri R, Monteiro CA, Mondini L. Distribuição regional e socioeconômica da disponibilidade domiciliar de alimentos no Brasil em 2008-2009. Rev Saúde Pública [serial on the internet]. 2012 [cited 2017 May 9];46(1):615. Available from: http://www.scielo.br/pdf/rsp/ v46n1/3014.pdf

4. Instituto Brasileiro de Geografia e Estatística. Pesquisa Nacional por Amostra de Domicílios - PNAD 2013 [document on the internet]. 2014 [cited 2017 May 27]. Available from: https://ww2.ibge.gov.br/ home/estatistica/pesquisas/pesquisa resultados. php?id pesquisa $=40$ 
5. Iulino BA, Mancuso AMC, Gambardella AMD. Educação nutricional em escolas de Ensino Fundamental do Município de Guarulhos-SP. Mundo Saúde [serial on the internet]. 2009 [cited 2017 May 7];33(3):264-72. Available from: http://www. saocamilo-sp.br/pdf/mundo saude/69/264a272.pdf

6. Brasil. Política Nacional de Alimentação e Nutrição [document on the internet]. 2013 [cited 2017 Aug 20]. Available from: http://bvsms.saude. gov.br/bvs/publicacoes/pnan.pdf

7. Prado BG, Guimarães LV, Lopes MAL, Bergamaschi DP. Efeito de ações educativas no consumo de alimentos no ambiente escolar. Nutrire (Online) [serial on the internet]. 2012 [cited 2017 May 5];37(3):281-92. Available from: http://docs. bvsalud.org/biblioref/2017/05/322082/artigo.pdf

8. Cavalcanti LA, Carmo Júnior TR, Pereira LA, Asano RY, Garcia MCL, Cardeal CM, et al. Efeitos de uma intervenção em escolares do Ensino Fundamental I, para a promoção de hábitos alimentares saudáveis. Rev Bras Ciênc Mov [serial on the internet]. 2012 [cited 2017 May 3];20(2):5-13. Available from: http://cev.org.br/biblioteca/efeitos-umaintervencao-escolares-ensino-fundamental-l-parapromocao-habitos-alimentares-saudaveis

9. Boog MCF. Programa de educação nutricional em escola de Ensino Fundamental de zona rural. Rev Nutr [serial on the internet]. 2010 [cited 2017 Mar 20];23(6):1005-17. Available from: http://www. scielo.br/pdf/rn/v23n6/07.pdf

10. Barbosa NVS, Machado NMV, Soares MCV, Pinto ARR. Alimentação na escola e autonomia: desafios e possibilidades. Ciênc Saúde Colet [serial on the internet]. 2013 [cited 2017 May 5];18(4):93745. Available from: http://www.scielo.br/pdf/csc/ v18n4/05.pdf

11. Azeredo OEA, Sá SPC, Lavoyer CE. Ações em educação nutricional com crianças em creche universitária: percepção dos responsáveis e dos professores sobre o lúdico. Rev Pesqui Cuid Fundam (Online) [serial on the internet]. 2014 [cited 2016 Jul 10];6(4):1419-36. Available from: http://seer. unirio.br/index.php/cuidadofundamental/article/ view $/ 3106 / p d f 734$

12. Anzolin C, Ouriques CM, Höfelmann DA, Mezadri T. Intervenções nutricionais em escolares. Rev Bras Pesqui Saúde [serial on the internet]. 2010 [cited 2017 May 3];23(4):297-306. Available from: https:// periodicos.unifor.br/RBPS/article/view/2030/2325

13. Piccoli L, Johann R, Corrêa EN. A educação nutricional nas séries iniciais de escolas públicas estaduais de dois municípios do oeste de Santa Catarina. Nutrire [serial on the internet]. 2010 [cited 2017 May 7];35(3):1-15. Available from: http://sban. cloudpainel.com.br/files/revistas publicacoes/295. $\underline{p d f}$
14. Juzwiak CR, Castro PM, Batista SHS. A experiência da Oficina Permanente de Educação Alimentar e em Saúde (OPEAS): formação de profissionais para a promoção da alimentação saudável nas escolas. Ciênc Saúde Colet [serial on the internet]. 2013 [cited 2017 Apr 14];18(4):1009-18. Available from: http://www.scielo.br/pdf/csc/v18n4/14.pdf

15. Prado BG, Fortes ENS, Lopes MAL, Guimarães LV. Ações de educação alimentar e nutricional para escolares: um relato de experiência. Demetra: Alimentação, Nutrição \& Saúde [serial on the internet]. 2016 [cited 2017 May 7];11(2):369-82. Available from: https://www.e-publicacoes.uerj.br/ index.php/demetra/article/view/16168/17722

16. Brasil. Guia alimentar para a população brasileira [document on the internet]. 2015 [cited 2017 Aug 20]. Available from: http://bvsms.saude.gov. br/bvs/publicacoes/guia alimentar populacao brasileira 2ed.pdf

17. Albuquerque AG, Cerqueira MMO. Conhecimentos e práticas de educadores e nutricionistas sobre a educação alimentar e nutricional no ambiente escolar [document on the internet]. 2012 [cited 2019 May 27]. Available from: https://repositorio.ufpe.br/ bitstream/123456789/9087/1/arquivo9584 1.pdf

18. Maia ER, Lima Junior JF, Pereira JS, Eloi AC, Gomes CC, Nobre MMF. Validação de metodologias ativas de ensino-aprendizagem na promoção da saúde alimentar infantil. Rev Nutr [serial on the internet]. 2012 [cited 2017 Feb 22];25(1):79-88. Available from: http://www.scielo.br/pdf/rn/v25n1/ a08v25n1.pdf

19. Câmara Interministerial de Segurança Alimentar e Nutricional. Estratégia intersetorial de prevenção e controle da obesidade: recomendações para estados e municípios [document on the internet]. 2014 [cited 2017 Aug 20]. Available from: http://www.mds.gov. br/webarquivos/publicacao/seguranca alimentar/ estrategiaobesiddade.pdf

20. Lira RCM, Antunes LR, Mesquita K0. Experiência de abordagem sobre alimentação em crianças. Sanare (Sobral, Online) [serial on the internet]. 2016 [cited 2018 Mar 8];15(1):69-73. Available from: https://sanare.emnuvens.com.br/sanare/article/ view $/ 930 / 559$
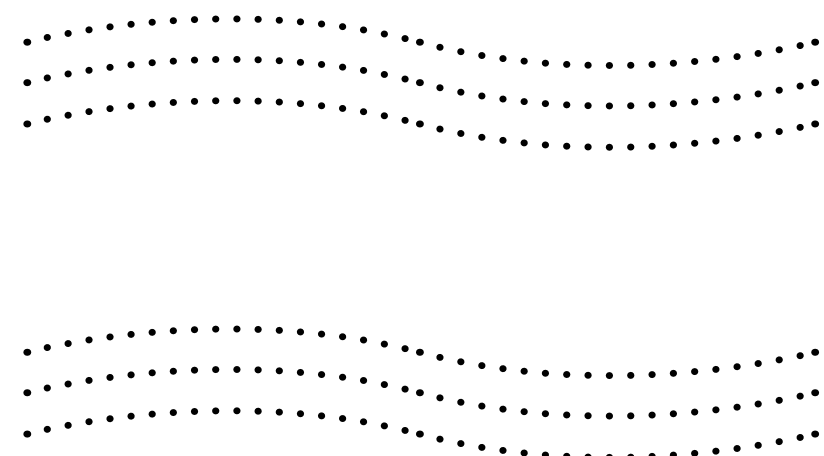